\title{
Prevalencia de fisura labiopalatina e indicadores de riesgo: Estudio de la población atendida en el Hospital Clínico Félix Bulnes de Santiago de Chile*
}

\author{
Prevalence of cleft lip and palate and risk indicators: Study of the reference \\ population of Felix Bulnes University Hospital, Santiago de Chile
}

\author{
G. Sepúlveda Troncoso' , H. Palomino Zúñiga², J. Cortés Araya ${ }^{1-3}$
}

\begin{abstract}
Resumen: Introducción. El estudio de la prevalencia de fisuras labiopalatinas y la determinación de indicadores de riesgo ayuda a prevenir su ocurrencia mejorando las condiciones durante la concepción o gestación. También favorece diagnósticos precoces que atenúan el impacto emocional favoreciendo el apego madre-hijo y mejorando la respuesta materna al tratamiento. Ambas situaciones permiten optimizar y focalizar los recursos sanitarios disponibles. Material y Método. Se determinó la incidencia de fisuras labiopalatinas y la asociación a factores de riesgo en los RNV beneficiarios del Hospital Clínico Félix Bulnes de Santiago de Chile entre enero del año 1998 y junio del 2005. Se estudió además condiciones y exposición maternas a agentes ambientales asociados a fisura labial y/o palatina. Resultados. Sobre un total de 36.041 RNV consecutivos, se registraron 51 casos de fisurados, obteniéndose una tasa de 1,42 x 1000 RNV. Conclusiones. Los indicadores de riesgo identificados correspondieron a edad materna menor a veinte años asociado al primer embarazo; alto grado de etnicidad amerindia e historia familiar positiva para este tipo de malformación. Nuestro estudio apoya la teoría de herencia multifactorial de la susceptibilidad a las fisuras labiopalatinas.
\end{abstract}

Palabras clave: Prevalencia; Fisuras faciales; Indicadores de riesgo.

Recibido: 09.10 .06

Aceptado: 11.01 .08

1 Docente. Departamento de Cirugía Bucal y Máxilofacial, Facultad de Odontología, Universidad de Chile.

2 Profesor Titular. Programa de Genética Humana, Instituto de Ciencias Biomédicas, Facultad de Medicina, Universidad de Chile.

3 Cirujano Máxilofacial. Unidad de Fisurados del Servicio de Cirugía Infantil, Hospital Clínico Félix Bulnes. Profesor Asociado del Departamento de Cirugía Bucal y Máxilofacial de la Facultad de Odontología de la Universidad de Chile.

*Trabajo realizado en la Unidad de Fisurados, Servicio de Cirugía Infantil, Hospital Clínico Félix Bulnes.

\section{Correspondencia:}

Juan Cortés Araya

Facultad de Odontología, Universidad de Chile

Av. Santa María 571, Santiago, Chile

Email: jcortes@uchile.cl

\begin{abstract}
Introduction. The study of the prevalence of cleft lip and palate and the identification of risk groups helps to improve the treatment of this condition. Occurrence may be prevented by improving environmental conditions during pregnancy and ensuring early diagnosis, thus lessening the emotional impact on the family and reinforcing the early mother-child bond. The aim of this study was to improve prevention levels and thus enhance health resources. Material and Method. The frequency of cleft lip and palate and its association with risk factors was studied in live births of the Felix Bulnes University Hospital in Santiago between January 1998 and June 2005. Maternal conditions and exposure to environmental agents associated with cleft lip and/or palate were examined. Results. Out of a total of 36,041 births, 51 cases of cleft lip and palate were found, which yielded a rate of $1.42 \times 1000$ births. Conclusions. Analysis of risk indicators identified: mothers under the age of 20 years, usually with their first pregnancy, a high degree of Amerindian admixture and family history positive for this malformation. Our study supports the multifactorial inheritance theory of susceptibility to cleft lip and palate.
\end{abstract}

Key words: Prevalence; Oral Clefts; Risk Factors. 


\section{Introducción}

Las fisuras de labio y/o paladar son malformaciones congénitas de alta prevalencia en Chile. Se estima que por cada 740 nacimientos de niños vivos hay un afectado con dicha condición. ${ }^{1}$ Esta comprobación epidemiológica representa un problema de salud pública.

La condición de fisurado desencadena en el paciente trastornos morfológicos, funcionales y emocionales que dificultan su inserción social. La familia y especialmente la madre, se ve comprometida emocionalmente. Este compromiso se desencadena al nacimiento, momento en que se informa del diagnóstico provocando un duelo que afecta la relación con el hijo. Por otra parte, el tratamiento de esta condición determina esfuerzos y gastos considerables. El estudio de la etiología y la determinación de indicadores de riesgo en poblaciones específicas mejoran la respuesta terapéutica. Por una parte en la prevención de la aparición, mejorando las condiciones durante la concepción y/o gestación y por otra consiguiendo diagnósticos precoces, eficaces en atenuar el impacto emocional materno, mejorando así el apego con su hijo y mejorando su disposición y participación en el tratamiento. Ambos objetivos buscan niveles de prevención que focalicen y optimicen los recursos sanitarios.

Existe evidencia que algunos factores genéticos determinan la aparición de fisuras. Existe un importante número de investigaciones que buscan determinar genes específicos, ${ }^{2-4}$ cómo se hereda, ${ }^{5,6}$ y las causas de las diferencias étnicas detectadas. ${ }^{7,8}$ También han sido identificados teratógenos que afectan directamente al embrión, tales como drogas y tóxicos de uso agrícola e industrial. ${ }^{9-13}$ Estas noxas también pueden afectar los gametos parentales y por ende al resto de su descendencia. La edad materna y enfermedades durante el embarazo parecieran jugar un rol en su patogénesis. ${ }^{14-17}$

El objetivo del presente trabajo es determinar la prevalencia de fisura labiopalatina en beneficiarios del Hospital Clínico Félix Bulnes (Chile) e indicadores de riesgo.

\section{Material y método}

Se examinaron 36.041 fichas correspondientes a todos los nacimientos ocurridos entre enero del año 1998 y junio del 2005 en el Hospital Clínico Félix Bulnes, Santiago (Chile), obteniendo 51 casos con diagnóstico de fisura de labio y/o paladar no sindromáticos. Otros 24 casos que correspondieron a derivaciones desde otros centros, fueron incluidos para el análisis de las variables, independientemente del lugar de nacimiento. Los antecedentes del niño y su madre fueron extraídos de sus historias clínicas. Se seleccionó al recién nacido vivo sano y del mismo sexo nacido inmediatamente después al paciente fisurado con el fin de establecer el grupo control, incluyendo la información de la madre.

La información se registró en una ficha especialmente diseñada que incluyó sexo, fecha de nacimiento, diagnóstico específico y grupo sanguíneo; mientras que en antecedentes de la madre se registró edad, orden de embarazo, recurrencia de malformaciones en parientes, consanguinidad parental, y exposición a teratógenos previo y durante el embarazo.

\section{Introduction}

Cleft lip and/or palate are congenital malformations with a high prevalence in Chile. One case per 740 live births is

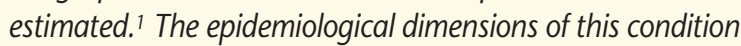
make it a public health problem.

The cleft condition originates morphologic, functional, and emotional disorders in the patient that make social insertion difficult. The family, particularly the mother, is affected emotionally. This emotional effect begins at birth, when the mother receives the diagnosis and her grief affects her relation with her newborn. On the other hand, treatment of this condition involves considerable effort and expenses. Study of the etiology and identification of risk indicators in specific populations could improve therapeutic response. Improving the conditions of conception and/or gestation may prevent occurrence. Early diagnosis may be effective in attenuating the emotional impact on the mother and improving her bond with her infant and disposition toward and participation in treatment. Both goals seek to achieve prevention levels that allow health care resources to be targeted and optimized.

Evidence exists that certain genetic factors condition the appearance of cleft lip and/or palate. Numerous studies have sought specific genes, $2-4$ form of inheritance,, 5 and causes of ethnic differences. ${ }^{7,8}$ Teratogens have been identified that directly affect the embryo, such as drugs and toxins used in agriculture and industry. ${ }^{9-13}$ These noxa may also affect parental gametes and the resulting offspring. Maternal age and diseases during pregnancy seem to contribute to pathogenesis. ${ }^{14-17}$

The aim of the present study was to determine the prevalence of cleft lip and/or palate in the reference population of the Felix Bulnes University Hospital (Chile) and to identify risk indicators.

\section{Material and method}

We examined the records of 36,041 births, corresponding to all live births between January 1998 and June 2005 in Felix Bulnes University Hospital, Santinago (Chile). We detected 51 infants diagnosed as nonsyndromic cleft lip and/or palate. Another 24 cases referred by other centers were included in the analysis of variables, independently of birthplace. The history of the case infants and their mothers was obtained from medical records. Case infants with cleft lip and/or palate were each paired with a healthy newborn of the same sex born immediately after the case infant for the purpose of establishing a control group; information on the mother of the healthy infant was also analyzed.

The information was recorded on a specially designed card that included gender, date of birth, specific diagnosis, and blood group. The maternal history included age, pregnancy order, occurrence of malformations in relatives, parent consanguinity, and exposure to teratogens before and during pregnancy. 
Para determinar el grado de etnicidad se obtuvieron las frecuencias fenotípicas de los grupos AB0 y Rh, estableciendo así el porcentaje de mezcla indígena, según el método de Bernstein. ${ }^{18}$

Los estudios de grado de mezcla se hicieron separadamente para el alelo $\mathrm{O}$ del grupo $\mathrm{ABO}$ y d del factor Rh negativo, fueron promediados y expresados como porcentaje de mezcla.

La información fue procesada y analizada estadísticamente mediante técnicas de cálculo de frecuencias, promedios, y análisis de chi cuadrado en las variables cualitativas.

\section{Resultados}

Para el estudio de prevalencia de fisura labiopalatina se obtuvieron 51 casos no sindrómicos de 36.041 nacimientos consecutivos entre enero de 1998 y junio de 2005. En la tabla 1 se muestra la distribución por sexo y diagnóstico. La fisura labiomáxilopalatina (FLMP) se dio en el $43,1 \%$ de los casos, seguido por $29,4 \%$ de fisura palatina aislada (FP) y $27,5 \%$ de fisura labial aislada (FL). Dentro del grupo FLMP, un $63,6 \%$ de los casos afectó el lado izquierdo y sólo 9,1\% el derecho. Seis casos, un 27,3\% fueron bilaterales. Entre las fisuras labiales, el 57,2\% correspondió al lado izquierdo, 35,7\% al derecho, y 7,1\% bilateral. Tanto FLMP como FL fueron más prevalentes en hombres, mientras que la FP lo fue en mujeres. La tasa de prevalencia total de fisura labiopalatina fue de 1,42 por 1.000 RNV. No hubo diferencia significativa en la distribución por género $\left(\mathrm{chi}^{2}=1,05 ; \mathrm{gl}=2 ; p=\right.$ $0,59)$.

La tabla 2 presenta el número de fisurados y las tasas por mil, separados por mes de nacimiento. La distribución entre meses es homogénea excepto por un considerable aumento en las tasas durante los meses de marzo y abril, con 3,03 y 3,19 por mil respectivamente, doblando aproximadamente la tasa total anual de 1,42 por 1000. Al ordenar por estaciones (Tabla 3), se observa una mayor tasa durante el otoño y una disminución de casos durante los meses de primavera (austral). Estas diferencias no
Tabla 2. $N^{\circ}$ y tasa $x 1000$ RNV fisurados por mes de nacimiento

$\begin{array}{lcc}\text { Mes } & \text { Total } & \text { Tasa por mes } \\ \text { Enero } & 6 & 2,00 \\ \text { Febrero } & 2 & 0,72 \\ \text { Marzo } & 10 & 3,03 \\ \text { Abril } & 9 & 3,19 \\ \text { Mayo } & 2 & 0,69 \\ \text { Junio } & 4 & 1,33 \\ \text { Julio } & 1 & 0,34 \\ \text { Agosto } & 5 & 1,81 \\ \text { Septiembre } & 5 & 1,61 \\ \text { Octubre } & 3 & 1,00 \\ \text { Noviembre } & 0 & - \\ \text { Diciembre } & 4 & 1,33 \\ \text { Total } & 51 & 1,42\end{array}$

Tabla 2. Number and rate $x 1000$ live births of cleft lip and/or palate per birth month

$\begin{array}{lcc}\text { Month } & \text { Total } & \text { Rate per month } \\ \text { January } & 6 & 2.00 \\ \text { February } & 2 & 0.72 \\ \text { March } & 10 & 3.03 \\ \text { April } & 9 & 3.19 \\ \text { May } & 2 & 0.69 \\ \text { June } & 4 & 1.33 \\ \text { July } & 1 & 0.34 \\ \text { August } & 5 & 1.81 \\ \text { September } & 5 & 1.61 \\ \text { October } & 3 & 1.00 \\ \text { November } & 0 & - \\ \text { December } & 4 & 1.33 \\ \text { Total } & 51 & 1.42\end{array}$
centage admixture.

The information was processed and analyzed statistically to calculate frequencies and averages. Chi square analysis was used with qualitative variables.

\section{Results}

A total of 51 nonsyndromic cases were detected in a universe of 36,041 consecutive births between January 1998 and June 2005 in the study of prevalence of cleft lip and/or palate. The distribution by gender and diagnosis is shown in table I. Cleft lip with maxillopalatine cleft (CLMP) occurred in $43.1 \%$ of cases, isolated cleft palate (CP) in $29.4 \%$, and isolated cleft lip (CL) in $27.5 \%$. Within the CLMP group, $63.6 \%$ of cases were of the left side and only $9.1 \%$ on the right side. Six cases (27.3\%) were bilateral. Among the cleft lips, $57.2 \%$ were on the left side, $35.7 \%$ on the right, and $7.1 \%$ were bilateral. CLMP and CL were both more prevalent in males, whereas CP was more prevalent in females. The overall prevalence of cleft lip and/or palate was 1.42 per 1000 live births. The distribution by gender disclosed no significant differences $\left(\right.$ Chi $\left.^{2}=1.05 ; d f=2 ; p=0.59\right)$. 
alcanzan significación estadística (chi ${ }^{2}=$ 7.37; $\mathrm{gl}=3 ; p=0.08$ ).

En la tabla 4 se observa la distribución por grupos sanguíneos $A B O$ y $R h$ en los sujetos fisurados, controles y sus madres. En todos los grupos, el alelo O fue el prevalente para el sistema $A B O$ con el $55,0 \%$ y el alelo $D(R h+)$ para el sistema Rh, con 99\% de casos.

De acuerdo a la presencia o no del alelo d del sistema Rhesus, la población estudiada ostentó un porcentaje de mezcla indígena de $52,9 \%$.

El rango de edades para madres de fisurados fue de 15 a 42 años, con un $36 \%$ dentro del grupo de 15 a 20 años y un descenso paulatino hasta el grupo de 41 a 45 años. En las madres controles, el rango de edad iba de 17 a 44 años. El grupo de madres control ubicado entre los 21 a 25 años fue el que exhibió un tamaño mayor, con el $36 \%$ de los casos. Se puede observar que el grupo de madres de afectados de edad entre los 15 a 20 años presentó un número mayor de individuos que su respectivo grupo control. Esta diferencia no resultó estadísticamente significativa $\left(\mathrm{chi}^{2}=9,61\right.$; $\mathrm{gl}=5 ; p=0,09$ ) (Tabla 5).

El orden de paridad en madres casos y controles y los intervalos entre embarazos, se exponen en la tabla 6 . El número de madres primerizas fue 30 y correspondió al $40 \%$; mientras que las primigestas controles resultaron sólo 14 , un $18.7 \%$, hecho estadísticamente significativo $\left(\mathrm{chi}^{2}=10,24\right.$; $\mathrm{gl}=4$ ). La mayor cantidad de madres de controles se concentra en el grupo de segundo y tercer embarazo, luego el número de madres comienza a descender a mayor orden de paridad, tal como ocurre en el grupo caso.

En la tabla 7 se expone el número y porcentaje de padres que informaron de recurrencia familiar de fisuras al momento de nacer, comparado con información aportada en meses posteriores. Se observa que el porcentaje de padres que conocía algún caso de fisura labiopalatina dentro de su familia aumenta si la entrevista se hace en meses o años posteriores al nacimiento. El $26,7 \%$ de los encuestados conocía por lo menos un afectado. palate

Tabla 3. Variación estacional en la incidencia de fisuras

\begin{tabular}{lcc} 
& \multicolumn{3}{c}{ Fisurados } \\
Estación & $\mathbf{N}^{\circ}$ & Tasa \\
Verano & 12 & 1,35 \\
Otoño & 21 & 2,30 \\
Invierno & 10 & 1,16 \\
Primavera & 8 & 0,87 \\
Total anual & 51 & 1,42 \\
$p=0,08$ & &
\end{tabular}

Tabla 3. Seasonal variation in the incidence of cleft lip and/or

\begin{tabular}{lcc} 
& \multicolumn{2}{c}{ Clefts } \\
Station & No & Rate \\
Summer & 12 & 1.35 \\
Autumn & 21 & 2.30 \\
Winter & 10 & 1.16 \\
Spring & 8 & 0.87 \\
Annual total & 51 & $\mathbf{1 . 4 2}$ \\
$p=0.08$ & &
\end{tabular}

Tabla 4. Distribución de grupos sanguíneos $A B O$ y $\mathrm{Rh}$

\begin{tabular}{lcccccc}
\multicolumn{4}{c}{} & \multicolumn{2}{c}{ Casos } & \multicolumn{2}{c}{ Controles } \\
Gijo & Madre & \multicolumn{2}{c}{ Hijo } & Madre & \multicolumn{2}{c}{ Total } \\
G. Sanguíneo & $\mathbf{N}^{\circ}$ & $\mathbf{N}^{\circ}$ & $\mathbf{N}^{\circ}$ & $\mathbf{N}^{\circ}$ & $\mathbf{N}^{\circ}$ & $\%$ \\
AB & 4 & 2 & 6 & 4 & 16 & 5,3 \\
A & 22 & 23 & 27 & 26 & 98 & 32,7 \\
B & 6 & 4 & 7 & 4 & 21 & 7 \\
O & 43 & 46 & 35 & 41 & 165 & 55,0 \\
Total & 75 & 75 & 75 & 75 & 300 & 100 \\
d (Rh-) & 1 & 1 & 0 & 1 & 3 & 1 \\
D (Rh+) & 74 & 74 & 75 & 74 & 297 & 99
\end{tabular}

Tabla 4. Distribution of $A B O$ and Rh blood groups

\begin{tabular}{lcccccc} 
& \multicolumn{2}{c}{ Cases } & \multicolumn{2}{c}{ Controls } & \\
Blood group & $\begin{array}{c}\text { Child } \\
\mathbf{N}^{\circ}\end{array}$ & $\begin{array}{c}\text { Mother } \\
\mathbf{N}^{\circ}\end{array}$ & $\begin{array}{c}\text { Child } \\
\mathbf{N}^{\circ}\end{array}$ & $\begin{array}{c}\text { Mother } \\
\mathbf{N}^{\circ}\end{array}$ & \multicolumn{2}{c}{ Total } \\
N & 4 & 2 & 6 & 4 & 16 & $\%$ \\
A & 22 & 23 & 27 & 26 & 98 & 32.7 \\
B & 6 & 4 & 7 & 4 & 21 & 7 \\
O & 43 & 46 & 35 & 41 & 165 & 55.0 \\
Total & 75 & 75 & 75 & 75 & 300 & 100 \\
d (Rh-)0 & 1 & 1 & 0 & 1 & 3 & 1 \\
D (Rh+) & 74 & 74 & 75 & 74 & 297 & 99
\end{tabular}

The number of infants with cleft lip and/or palate and rates per thousand, separated by birth month, are shown in Table II. The distribution by months was homogeneous except for a considerable increase in rates in March and April, to 3.03 and 3.19 per thousand, respectively. This rate was approximately twice the overall annual rate of 1.42 per 1000. The seasonal distribution (Table III) revealed a higher rate in autumn and a lower rate in spring (southern hemisphere). These differences were not statistically significant $\left(\right.$ chi $^{2}=7.37 ; d f=$ $3 ; p=0.08)$.

The distribution by $A B O$ and $R h$ blood groups of the case infants, control infants, and their mothers is shown in Table IV. The $O$ allele was prevalent for the $A B O$ system (55.0\%) and the $D$ allele $(R h+)$ was prevalent for the Rh system (99\%) in all groups. The presence or absence of the $d$ allele of the Rhesus system showed that the study population had $52.9 \%$ indigenous admixture.

The age range of the mothers of case infants was 15 to 42 years, with $36 \%$ in the 15 to 20-year age group and a progressively smaller percentage in other age ranges until the 41 to 45-year group.

The age range of the control mothers was 17 to 44 years. The largest group of control mothers was 21 to 25 years, with $36 \%$. The group of mothers age 15 to 20 years of case infants was larger than the respective group of control mothers. This difference was statistically nonsignificant $\left(\mathrm{chi}^{2}=\right.$ 9.61; $d f=5 ; p=0.09$ ) (Table V). 
En la tabla 8 se presenta los casos en que los padres estuvieron expuestos a agentes teratógenos previos y durante el embarazo. Nueve madres de fisurados relataron haber fumado durante el embarazo, versus tres del grupo control. Tal diferencia no alcanzó significación estadística debido al tamaño muestral. Sólo una madre de fisurado refirió haber ingerido alcohol durante su gravidez. Siete madres del grupo de casos y tres del grupo control ingirieron anticonceptivos orales durante las primeras semanas posteriores a la fecundación. Un total de 17 madres ingirió antiinflamatorios no esteroidales durante el embarazo como terapia de resfrío común y/o cefaleas. Dentro de este grupo, 13 correspondieron a madres de fisurados y 4 a controles. Por último, dos personas del grupo caso relataron haber sido irradiadas durante el embarazo, y seis expuestas laboralmente a agentes nocivos. Entre éstos últimos, cinco se ocupaban en labores relacionadas con productos agrícolas y en un caso ambos padres del niño afectado realizaban labores de aseo industrial.

Dentro de las patologías que afectaron a las madres durante el período de embarazo, 13 relataron haber sufrido estados de mucho estrés, secundario a embarazo no deseado o depresión crónica. La distribución resultó homogénea en ambos grupos, con 7 madres en el grupo de casos y 6 en el grupo control. Cinco embarazadas del grupo casos fueron afectadas por infecciones graves que necesitaron antibioterapia. Cinco madres caso presentaron hipertensión arterial, y cuatro diabetes mellitus o gestacional. Estos resultados se exponen en la tabla 9.
Tabla 6. Orden de parto y meses de intervalo entre embarazos

\begin{tabular}{lcccccc} 
& \multicolumn{3}{c}{ Casos } & \multicolumn{3}{c}{ Controles } \\
& $\mathbf{N}^{\circ}$ & $\%$ & Int & $\mathbf{N}^{\circ}$ & $\%$ & Int \\
Primero & 30 & 40,0 & & 14 & 18,7 & \\
$2^{\circ}$ embarazo & 22 & 29,3 & $59,4 \mathrm{~m}$ & 25 & 33,3 & $53,1 \mathrm{~m}$ \\
$3^{\circ}$ embarazo & 13 & 17.3 & $99 \mathrm{~m}$ & 25 & 33,3 & $53,8 \mathrm{~m}$ \\
$4^{\circ}$ embarazo & 5 & 6,7 & $30 \mathrm{~m}$ & 7 & 9,3 & $56 \mathrm{~m}$ \\
$5^{\circ}$ embarazo o más & 5 & 6,7 & $21 \mathrm{~m}$ & 4 & 5,3 & $61 \mathrm{~m}$ \\
Chín $^{2}$ prim emb= 10,24; gl= $4 ; p=0,04$. & & & & &
\end{tabular}

Tabla 6. Birth order and interval between pregnancies in months

\begin{tabular}{lccccccc} 
& \multicolumn{3}{c}{ Cases } & \multicolumn{3}{c}{ Controls } \\
& $\mathbf{N}^{\circ}$ & $\%$ & Int & $\mathbf{N}^{\circ}$ & $\%$ & Int \\
First & 30 & 40.0 & & 14 & 18.7 & \\
2nd pregnancy & 22 & 29.3 & $59,4 \mathrm{~m}$ & 25 & 33.3 & $53.1 \mathrm{~m}$ \\
3th pregnancy & 13 & 17.3 & $99 \mathrm{~m}$ & 25 & 33.3 & $53.8 \mathrm{~m}$ \\
4th pregnancy & 5 & 6,7 & $30 \mathrm{~m}$ & 7 & 9.3 & $56 \mathrm{~m}$ \\
5th pregnancy or more & 5 & 6.7 & $21 \mathrm{~m}$ & 4 & 5.3 & $61 \mathrm{~m}$ \\
Chi2 1st preg=10.24; $g l=4 ; p=0.04$. & & & & &
\end{tabular}

The birth order of the case and control mothers and intervals between pregnancies are shown in Table VI. Primiparity was found in 30 (40\%) case mothers as opposed to 14 (18.7\%) control mothers (chi2 $=10.24 ; d f=4)$. The index pregnancy was the second or third for many control mothers, and less frequently, the fourth pregnancy or more. This was also true among case mothers.

The number and percentage of parents who reported a familial incidence of cleft lip and/or palate when questioned at birth versus the number who gave this information in later months is summarized in Table VII. The percentage of parents aware of a familial case of cleft lip and/or palate increased in interviews held months or years after the index birth. The survey disclosed $26.7 \%$ of families with at least one other family member affected.

Cases of parents exposed to teratogens before and during pregnancy are analyzed in Table VIII. Nine case mothers versus 3 control mothers reported smoking during pregnancy. The difference was not statistically significant due to sample size. Only one case mother reported alcohol intake during pregnancy. Seven case mothers versus 3 control mothers took oral contraceptives in the first weeks after fertilization. A total of 17 mothers took nonsteroidal anti-inflammatory drugs during pregnancy for common colds and/or headache. This group contained 13 case mothers versus 4 control mothers. Finally, 2 case mothers reported being radiated during pregnancy and 6 were exposed to harmful agents during work. In the latter group, 5 worked de factores genéticos y ambientales predisponentes. 
La prevalencia de fisuras obtenida corresponde a 1,42 por $1000 \mathrm{RNV}$, esto significa 1 por 704 nacimientos aproximadamente, tasa similar al promedio nacional. ${ }^{1,20}$ La distribución por diagnóstico y sexo sigue los patrones esperados, con una relación aproximada de 1:2:1 para FL, FLMP y FP. En FL y FLMP los hombres son más afectados y el izquierdo el lado afectado. Previsiblemente la FP resulta más frecuente entre mujeres. En la fisura de labio y paladar primario, el lado más afectado es el izquierdo, aunque dentro del grupo FLMP la diferencia entre lados es mayor a lo esperado.

Hemos observado que existe variación estacional en la aparición de fisuras con una tasa mayor en otoño, correspondiente a concepciones ocurridas durante el invierno, meses en que existe predisposición a infecciones de la vía respiratoria asociada con ingesta de antiinflamatorios y antibióticos. No obstante, esta diferencia no alcanza significación estadística probablemente debido al tamaño de la muestra.. Investigaciones análogas realizadas en otras zonas de Santiago, ${ }^{21}$ sí las han confirmado.

El Hospital estudiado brinda atención a personas de nivel socioeconómico medio y bajo, estratos que han sido relacionados con un mayor grado de etnicidad amerindia y por esto con probables tasas mayores de fisuras del labio y paladar. 7,22

El estudio de la etnicidad amerindia, obtenido por la frecuencia génica del alelo $O$ y de los sistemas $A B O$ y Rh, confirma que existe un porcentaje de etnicidad amerindia importante en los sujetos estudiados existiendo una correlación casi completa entre grado de etnicidad amerindia y tasas de prevalencia. Hemos obtenido el mayor porcentaje de etnicidad amerindia dentro de los estudios publicados, ${ }^{23,24}$ lo que se correlaciona con la alta frecuencia de fisuras. Sin embargo, en localidades emplazadas en altura como Chuquicamata y Calama, es posible observar tasas de fisuras mayores, aunque su índice de indigenicidad es menor. ${ }^{23}$ Este hecho reafirma la importancia de los factores ambientales en la aparición de fisuras, respaldando la teoría de una etiología multifactorial.

Coincidente a otros trabajos, encontramos que la fisura labial es más frecuente en varones y la fisura palatina en mujeres. Igualmente,
Tabla 7. Conocimiento por parte de los padres de recurrencia familiar de fisuras según el tiempo de entrevista

\begin{tabular}{|c|c|c|}
\hline & $\mathbf{N}^{\circ}$ & $\%$ \\
\hline \multicolumn{3}{|c|}{ Al momento del nacimiento } \\
\hline Sabe que existe & 14 & 18,7 \\
\hline No sabe & 61 & 81,3 \\
\hline \multicolumn{3}{|c|}{ Con posterioridad } \\
\hline Sabe que existe & 20 & 26,7 \\
\hline No sabe & 55 & 73,3 \\
\hline
\end{tabular}

Tabla 7. Knowledge of parents of family members with cleft lip and/or palate depending on time of interview

$\begin{array}{llc} & \mathrm{N}^{\circ} & \% \\ \text { At time of birth } & & \\ \text { Aware } & 14 & 18.7 \\ \text { Unaware } & 61 & 81.3 \\ \text { Con posterioridad } & & \\ \text { Aware } & 20 & 26.7 \\ \text { Unaware } & 55 & 73.3\end{array}$

Tabla 8. Exposición a agentes teratógenos

$\begin{array}{lcc} & \text { Casos } & \text { Controles } \\ \text { Tabaco } & 9 & 3 \\ \text { Anticonc. Orales } & 7 & 3 \\ \text { AINEs } & 13 & 4 \\ \text { Otros } & 9 & -\end{array}$

Tabla 8. Exposure to teratogenic agents

$\begin{array}{lcc} & \text { Cases } & \text { Controls } \\ \text { Tobacco } & 9 & 3 \\ \text { Oral contrac. } & 7 & 3 \\ \text { NSAIDs } & 13 & 4 \\ \text { Others } & 9 & -\end{array}$

Tabla 9. Enfermedades maternas durante el embarazo

$\begin{array}{lcc} & \text { Casos } & \text { Controles } \\ \text { Estrés } & 7 & 6 \\ \text { Inf. Agudas } & 5 & \\ \text { HTA } & 5 & \\ \text { DM } & 4 & 1\end{array}$

Tabla 9. Maternal diseases during pregnancy

$\begin{array}{lcc} & \text { Cases } & \text { Controls } \\ \text { Stress } & 7 & 6 \\ \text { Acute inf. } & 5 & \\ \text { AHT } & 5 & \\ \text { DM } & 4 & 1\end{array}$
often affected is the left side, although the difference between sides is greater than expected in the CLMP group. with agricultural products and in one instance both case parents had contact with industrial cleaning products.

The pathologies that affected mothers during pregnancy included 13 mothers who reported stress due to an unwanted pregnancy or chronic depression. The distribution was similar in both groups with 7 case mothers and 6 control mothers. Five case mothers had serious infections that required antibiotic therapy. Five case mothers had arterial hypertension and 4 had diabetes mellitus or gestational diabetes. These results are summarized in table IX.

\section{Discussion}

Cleft lip and/or palate are the most frequent craniofacial malformations in the world. In Chile this malformation is an important problem because of its prevalence and the consequences for the patient and the patient's circle. ${ }^{19}$

Many studies have been made to clarify the etiology of this conditions, but no cause has been identified, only a series of predisposing genetic and environmental factors.

The prevalence of cleft lip and/or palate in our study was 1.42 per 1000 live births, or approximately 1 out of 704 births, a rate similar to the national average. ${ }^{1,20}$ The distribution by diagnosis and sex coincides with the expected patterns, with an approximate ratio of 1:2:1 for CL, CLMP, and $C P$. CL and CLMP affected males and the left side more often. CP was more frequent among females. In primary cleft lip and/or palate, the side most 
tanto en varones como en mujeres, en la fisura labial unilateral el lado mayormente afectado es el izquierdo, aunque no existe explicación para ello.

El $40 \%$ de las madres de fisurados resultaron primigestas, y en ellas el $36 \%$ pertenecen al grupo de menor edad.

Otro factor importante en la susceptibilidad a fisuras es la edad materna. Algunos indican que las madres con riesgo mayor son las mayores de 35 años. ${ }^{14,25}$ Sin embargo, nuestro estudio muestra un número mayor de madres menores de 20 años. Esto está en relación directa con el número de madres primigestas, las que en nuestro estudio resultaron más numerosas en el grupo de casos que en el de controles. En Chile, en las dos últimas décadas ha habido un aumento progresivo del número de embarazos en menores de 19 años (INE). En el embarazo adolescente de la población estudiada confluyen múltiples indicadores de riesgo, a saber, pobreza, déficit educacional, paro, consumo de drogas y de alcohol entre otras. Desde esta perspectiva es factible imaginar que una intervención focalizada en este segmento lo beneficiará en su conjunto, incluyendo una disminución en la aparición de fisuras orales.

En Chile existe una alta recurrencia familiar de fisuras. ${ }^{6}$ En nuestro estudio el $26,7 \%$ de las familias encuestadas conoce algún fisurado en su genealogía. Es importante destacar la importancia del momento en que se realiza la encuesta, ya que la madre no cuenta durante los primeros días de vida del hijo con suficiente información de su familia o de la del padre. Al realizar el interrogatorio pasado algunos meses del nacimiento normalmente ella ha investigado y dispone de antecedentes. De hecho en nuestro estudio, seis personas encontraron parientes afectados que previamente no conocían.

Han sido identificados distintos factores ambientales como posibles causantes de la malformación. Destaca el humo del tabaco, generalmente asociado a la ingesta de alcohol y otros tóxicos. En nuestro estudio hemos comprobado que una parte de la población fuma durante el embarazo. Comparativamente existen más fumadoras en el grupo de madres de fisurados que de controles. ${ }^{11}$

Otro agente ingerido durante el embarazo fueron anticonceptivos orales. En estos casos la conciencia de estar embarazada fue tardío por lo que siguió con el consumo en promedio, durante los dos primeros meses de gestación. Es importante señalar que Peterson, ${ }^{27}$ y Bracken, ${ }^{28}$ asociaron el uso de anticonceptivos orales con malformaciones congénitas.

Otro indicador de riesgo correspondió a exposición laboral de padres a agentes químicos. Cabe mencionar que de 6 casos de exposición a químicos 5 de ellos correspondieron a padres que se desempeñaban en labores agrícolas en períodos pre y post concepcional. Esto concuerda con los estudios de Gordon y Shy, ${ }^{29}$ y Croen y cols. ${ }^{30}$ y además se relaciona con la intensiva industrialización agrícola de la zona central de Chile.

Las condiciones patológicas de la madre embarazada o su tratamiento suelen afectar el desarrollo intrauterino del hijo. El estrés observado en mujeres embarazadas, relacionado en la mayoría de los casos a embarazos no deseados, es una condición frecuente entre madres adolescentes. Como lo sugiere Palomino y cols., ${ }^{24}$ algunas anomalías psicológicas podrían jugar un rol importante en la aparición de fisuras orales. Ésta habitualmente se asocia al con-
We observed a seasonal variation in the occurrence of this malformation, with a higher rate in autumn, corresponding to children conceived in winter. In winter, there is a predisposition to respiratory tract infections associated with the use of anti-inflammatory agents and antibiotics. This difference is not statistically significant, probably due to the sample size. Similar studies in other areas of Santiago ${ }^{21}$ confirm this finding.

The reference population of the study hospital is of low to medium socioeconomic level, strata that are related with a greater degree of Amerindian extraction and probably with higher rates of cleft lip and/or palate. 7,22

The study of Amerindian ethnicity by the frequency of the $O$ allele and the $A B O$ and $R h$ systems confirmed a high percentage of Amerindian ethnicity among the study subjects. There was an almost complete correlation between degree of Amerindian admixture and prevalence rates. We obtained the highest percentage of Amerindian ethnicity among published studies, 23,24 which correlated with the high frequency of cleft lip and/or palate. However, in high altitude locations like Chuquicamata and Calama, higher rates of cleft lip and/or palate are observed, although the percentage indigenous extraction is lower. ${ }^{23}$ This finding confirms the importance of environmental factors in the appearance of cleft lip and/or palate, thus supporting the hypothesis of a multifactorial origin.

As in other studies, we found that cleft lip was more frequent in males and cleft palate was more frequent in females. In males and females, unilateral cleft lip affects mainly the left side, which remains unexplained.

Of the case mothers, $40 \%$ were primigravid and $36 \%$ belonged to the youngest age group.

Another important factor in susceptibility to cleft lip and/or palate is maternal age. Some studies indicate that the mothers at greatest risk are older than 35 years. ${ }^{14,25}$ However, our study found more mothers under the age of 20 years. This is directly related with the number of primigravid mothers, which in our study were more numerous in the case group than in the control group. In Chile, there has been a progressive increase in the number of pregnancies in women under 19 years in the last two decades (INE). Multiple risk factors converge in the adolescent pregnancies of the study population, e.g., poverty, low educational level, unemployment, and alcohol and drug use, among others. From this perspective, it is likely that any intervention targeting this segment will benefit the group as a whole, perhaps reducing the incidence of oral clefts.

There is a high familial incidence of cleft lip and/or palate in Chile. 6 In our study $26.7 \%$ of the families surveyed knew of a case among their relatives. It is important to emphasize the importance of the time when the survey is made because the mother often lacked information about her own family or that of the father in the first days of her child's life. When the survey was made a few months after birth, the mother usually had made inquiries and knew her family history. In 
sumo de tranquilizantes, los cuales han sido comprobados como teratógenos. ${ }^{31}$

Es sabido también que patologías crónicas como diabetes mellitus (DM) e hipertensión arterial (HTA) son factores de riesgo para tener hijos malformados. En nuestro estudio se observó que dentro de las madres de fisurados, cinco de ellas presentaron HTA durante el embarazo y cuatro DM. Se postula que en el caso de la hipertensión ocurriría una disminución precoz del flujo uteroplacentario, y en la diabetes el ambiente hiperglicémico actuaría como teratógeno. ${ }^{16}$

\section{Conclusiones}

Los indicadores de riesgo para malformaciones del labio y/o paladar entre los beneficiarios del Hospital Clínico Félix Bulnes son:

- Primigestas menores de veinte años.

- Proporción amerindia importante e historia familiar de malformaciones del labio y/o paladar.

Existe un riesgo aumentado en:

- Embarazadas que fuman, beben alcohol o ingieren medicamentos tipo AINEs o anticonceptivos orales y/o están expuestas a radiaciones.

- Padres que trabajan con productos químicos agrícolas y madres con patologías crónicas como hipertensión arterial y diabetes mellitus o que hayan pasado por períodos importantes de estrés en el embarazo temprano o con algún cuadro infeccioso grave. Nuestro estudio apoya la teoría de herencia multifactorial de la susceptibilidad a las fisuras labiopalatinas.

José Luis López Cedrún

Servicio de Cirugía Oral y Maxilofacial Hospital Universitario Juan Canalejo. La Coruña. España

\section{Bibliografía}

1. Protocolo AUGE, fisura labiopalatina para niños. Disponible en: www.minsal.cl (Consultado en octubre del 2004).

2. Blanco R, Suazo J, Santos J, Paredes M, Sung H, Carreño H, Jara L. Association between 10 microsatellite markers and nonsyndromic cleft lip palate in the chilean population. Cleft Palate Craniofac J 2004;41:163-7.

3. Blanco R, Suazo J, Santos J, Carreño H, Paredes M, Jara L, Eltit G. Evaluación de la asociación entre marcadores de microsatélite en 6p22-25 y fisura labiopalatina no sindrómica utilizando el diseño de tríos caso-progenitores en la población chilena. Rev Med Chile 2003;131:765-72.

4. Blanco R, Suazo J, Santos J, Carreño H, Paredes M, Jara L. Cleft Palate Craniofac I 2005;42: 267-71.

5. Palomino H, Cerda-Flores R, Blanco R, Palomino HM, Barton S, De Andrade M, Chakraborty R. Complex segregation analysis of facial clefting in Chile. J Craniofac Genet Dev Biol 1997; 17:57-64.

6. Palomino H, Guzmán E, Blanco R. Recurrencia familiar de labio leporino con o sin fisura velopalatina de origen no sindrómico en poblaciones de Chile. Rev Med Chile 2000;128:286-93. our study, 6 women found previously affected relatives that they had been unaware of.

Different environmental factors have been identified as possible causes of the malformation. Tobacco smoke, generally associated with ingestion of alcohol and other toxins, are noteworthy. We found that part of the population smoked during pregnancy in our study. More case mothers than control mothers were smokers. ${ }^{11}$

Oral contraceptives were another agent used during pregnancy. In these cases the mother failed to realize that she was pregnant and continued taking contraceptives for an average of two months of pregnancy. It is important to note that Peterson ${ }^{27}$ and Bracken ${ }^{28}$ have associated the use of oral contraceptives with congenital malformations.

Another risk factor was occupational exposure of the parents to chemical agents. The fact that 5 out of 6 cases of chemical exposure involved parents who worked on farms before and after conception merits mention. This coincides with the findings of Gordon and Shy ${ }^{29}$ and Croen et a ${ }^{30}$ and is related to the intensive industrialization of farming in central Chile.

Pathological conditions of the pregnant mother or their treatment usually affect the intrauterine development of the infant. The stress of pregnant women, generally related with an unwanted pregnancy, is a common condition among adolescent mothers. As Palomino et al24 suggest, some mental disorders may play an important role in the appearance of cleft lip and/or palate. For instance, it is often associated with tranquilizer use, which is a confirmed teratogen. ${ }^{31}$

It also is known that chronic pathologies like diabetes mellitus (DM) and arterial hypertension (AHT) are risk factors for malformations in offspring. Among the case mothers, 5 women had AHT during pregnancy and 4 had DM. It is postulated that there would be an early reduction of uteroplacental blood flow in hypertension and that the hyperglycemic medium would be teratogenic in diabetes. ${ }^{16}$

\section{Conclusions}

Risk factors for malformations of the lip and/or palate among the reference population of the Felix Bulnes University Hospital were:

- First pregnancy in women younger than 20 years old.

- Large proportion of Amerindian origin and family history of malformations of the lip and/or palate.

Risk was greater in:

- Pregnant women who smoked, drank alcohol, or took NSAIDs or oral contraceptives and/or were exposed to radiation.

- Parents who worked with agricultural chemicals, mothers with chronic pathologies like arterial hypertension and diabetes mellitus, or mothers who suffered important periods of stress in early pregnancy or a serious infection.

Our study supports the multifactorial heredity hypothesis of susceptibility to cleft lip and/or palate. 
7. Vanderas A. Incidence of cleft lip, cleft palate, and cleft lip and palate among races: A review. Cleft Palate / 1987;24:216-25.

8. Palomino HM, Palomino H, Cauvi D, Barton SA, Chakraborty R. Facial clefting and amerindian admixture in populations of Santiago, Chile. Am J Hum Biol 1997; 9:225-32.

9. Rojas A, Ojeda ME, Barraza X. Malformaciones congénitas y exposición a pesticidas. Rev Med Chile 2000;128:399-404.

10. Shaw G, Nelson V, lovannisci D, Finnell R, Lammer E. Maternal occupational chemical exposures and biotransformation genotypes as risk factors for selected congenital anomalies. Am J Epidemiol 2003;157:475-84.

11. Wyszynski D, Duffy D, Beaty T. Maternal cigarrete smoking and oral clefts: a meta-analysis. Cleft Palate Craniofac / 1997;34:206-10.

12. Lorente C, Cordier S, Goujard J, Aymé S, Bianchi F, Calzolari E, De Walle $\mathrm{H}$, Knill-jones R. Tobacco and alcohol use during pregnancy and risk of oral clefts. Occupational exposure and congenital malformation working group. Am J Public Health 2000;90:415-9.

13. Källén B. Maternal drug use and infant cleft lip/palate with special reference to corticoids. Cleft Palate Craniofac J 2003;40:624-8.

14. Nazer J, Cifuentes L, Ruiz G, Pizarro M. Edad materna como factor de riesgo para malformaciones congénitas. Rev Med Chile 1994;122: 299-303.

15. Pardo R, Nazer J, Cifuentes L. Prevalencia al nacimiento de malformaciones congénitas y de menor peso de nacimiento en hijos de madres adolescentes. Rev Med Chile 2003;131:1165-1172.

16. Ordoñez M, Nazer J, Aguila A, Cifuentes L. Malformaciones congénitas y patología crónica de la madre. Estudio ECLAMC 1971-1999. Rev Med Chile 2003; 131:404-11.

17. Vieira A, Orioli I, Murray J. Maternal age and oral clefts: A reappraisal. Oral Surg Oral Med Oral Pathol Oral Radiol Endod 2002;94:530-5.

18. Bernstein F. Die geographische Verteilung der Blutgruppen und ihre anthropologische Bedeutung (pp 227-243) en Comitato Italiano per lo studio del problemi della populazione. Instituto Poligrafico dello stato: Rome 1931.18.

19. Cobourne M. The complex genetics of cleft lip and palate. Eur / Orth 2004;26: 7-16.
20. Nazer H, Hubner G, Catalan M, y cols. Incidencia de labio leporino y paladar hendido en la Maternidad del Hospital Clínico de la Universidad de Chile y en las maternidades chilenas participantes en el Estudio Colaborativo Latino Americano de Malformaciones Congénitas (ECLAMC) período 1991-1999. Rev Med Chile 2001;129:285-93.

21. Blanco R, Cifuentes L, Muñoz MA, Fuchslocher G, Cauvi D, Arancibia $X$. Fisuras labio-palatinas en Santiago de Chile: Estudio epidemiológico. Rev Med Chile 1988;116:1320-6.

22. Valenzuela C, Acuña M, Harb Z. Gradiente sociogenético en la población chilena. Rev Med Chile 1987;115:295-9.

23. Goycoolea A, Palomino HM, Palomino H, Blanco R. Agentes medioambientales en la susceptibilidad a las fisuras faciales en el Norte de Chile. Odont Chilena 1993;41:113-9.

24. Palomino H, Palomino HM, Goycoolea A. Correlación de la frecuencia de fisuras faciales con atributos socio-genéticos y del medio ambiente en Chile. An Acad A Leng 1991;9:16-24.

25. Vieira A, Orioli I. Birth order and oral clefts: a meta analysis. Teratology 2002; 66:209-16.

26. Smits L, Essen G. Short interpregnancy intervals and unfavourable pregnancy outcome: role of folate depletion. Lancet 2001;358:2074-2077.

27. Peterson W. Pregnancy following oral contraceptive therapy. Obstetric and Gynecology 1969;34:363-7.

28. Bracken M. Oral contraception and congenital malformations in offspring: a review and mata-analysis of the prospective studies. Obstetric and Gynecology 1990;276:552-7.

29. Gordon J, Shy C. Agricultural chemical use and congenital cleft lip and/or cleft palate. Arch Environ Health 1981;36:213-21.

30. Croen L, Shaw G, Sanbonmatsu L, Selvin S, Buffler P. Maternal residential proximity hazardous waste sites and risk for selected congenital malformations. Epidemiology 1997;8:347-54.

31. Dolovich L, Addis A, Vaillancourt J, Barry J, Koren G, Einarson T. Benzodiazepine use in pregnancy and major malformations or oral cleft: meta-analysis of cohort and case-control studies. BMJ 1998;317:83943. 